\title{
Asymptotic behaviour of exponential functionals of Lévy processes with applications to random processes in random environment
}

\author{
Sandra Palau, Juan Carlos Pardo and Charline Smadi \\ Centro de Investigación en Matemáticas A.C. \\ Calle Jalisco s/n. 36240 Guanajuato, México. \\ E-mail address: \{ sandra.palau , jcpardo \} @cimat.mx \\ LISC-Laboratoire d'Ingénierie des Systèmes Complexes, IRSTEA \\ 9 avenue Blaise Pascal, 63178 Aubière, France \\ Department of Statistics, University of Oxford, \\ 24-29 St Giles', Oxford, OX1 3LB, UK \\ E-mail address: charline.smadi@polytechnique.edu
}

Abstract. Let $\xi=\left(\xi_{t}, t \geq 0\right)$ be a real-valued Lévy process and define its associated exponential functional as follows

$$
I_{t}(\xi):=\int_{0}^{t} \exp \left\{-\xi_{s}\right\} \mathrm{d} s, \quad t \geq 0 .
$$

Motivated by applications to stochastic processes in random environment, we study the asymptotic behaviour of

$$
\mathbb{E}\left[F\left(I_{t}(\xi)\right)\right] \quad \text { as } \quad t \rightarrow \infty,
$$

where $F=(F(x), x \geq 0)$ is a function with polynomial decay at infinity and which is non increasing for large $x$. In particular, under some exponential moment conditions on $\xi$, we find five different regimes that depend on the shape of the Laplace exponent of $\xi$. Our proof relies on a discretization of the exponential functional $I_{t}(\xi)$ and is closely related to the behaviour of functionals of semi-direct products of random variables.

We apply our results to three questions associated to stochastic processes in random environment. We first consider the asymptotic behaviour of extinction and explosion for self-similar continuous state branching processes in a Lévy random environment. Secondly, we focus on the asymptotic behaviour of the mean population size in a model with competition or logistic growth which is affected by a Lévy random environment and finally, we study the tail behaviour of the maximum of a diffusion in a Lévy random environment.

Received by the editors June 21, 2016; accepted November 30, 2016.

2010 Mathematics Subject Classification. 60G17, 60G51, 60G80.

Key words and phrases. Lévy processes, exponential functional, continuous state branching processes in random environment, explosion and extinction probabilities, logistic process, diffusions in random environment. 


\section{Introduction and main results}

A one-dimensional Lévy process is a stochastic process issued from the origin with stationary and independent increments and almost sure càdlàg paths. We write $\xi=\left(\xi_{t}: t \geq 0\right)$ for its trajectory and $\mathbb{P}$ for its law. The process $\xi$ is a strong Markov process, and for each $x \in \mathbb{R}$, we denote by $\mathbb{P}_{x}$ its law when issued from $x$ with the understanding that $\mathbb{P}_{0}=\mathbb{P}$. The law of a Lévy process is characterized by its one-time transition probabilities. In particular there always exists a triple $(\delta, \rho, \Pi)$ where $\delta \in \mathbb{R}, \rho \in \mathbb{R}$ and $\Pi$ is a measure on $\mathbb{R} \backslash\{0\}$ satisfying the integrability condition $\int_{\mathbb{R}}\left(1 \wedge x^{2}\right) \Pi(\mathrm{d} x)<\infty$, such that, for all $z \in \mathbb{R}$

$$
\mathbb{E}\left[e^{i z \xi_{t}}\right]=e^{t \psi(i z)},
$$

where the Laplace exponent $\psi(z)$ is given by the Lévy-Khintchine formula

$$
\psi(z)=\frac{1}{2} \rho^{2} z^{2}+\delta z+\int_{\mathbb{R}}\left(e^{z x}-1-z x \ell(x)\right) \Pi(\mathrm{d} x), \quad z \in \mathbb{R} .
$$

Here, $\ell(x)$ is the cutoff function which is usually taken to be $\ell(x)=\mathbf{1}_{\{|x|<1\}}$. Whenever the process $\xi$ has finite mean, we will take $\ell(x) \equiv 1$.

In this paper, we are interested in studying the exponential functional of $\xi$, defined by

$$
I_{t}(\xi):=\int_{0}^{t} e^{-\xi_{s}} \mathrm{~d} s, \quad t \geq 0
$$

In recent years there has been a general recognition that exponential functionals of Lévy processes play an important role in various domains of probability theory such as self-similar Markov processes, generalized Ornstein-Uhlenbeck processes, random processes in random environment, fragmentation processes, branching processes, mathematical finance, Brownian motion on hyperbolic spaces, insurance risk, queueing theory, to name but a few (see Bertoin and Yor, 2005; Carmona et al., 1997; Kyprianou and Pardo, 2008 and references therein).

There is a vast literature about exponential functionals of Lévy processes drifting to $+\infty$ or killed at an independent exponential time $\mathbf{e}_{q}$ with parameter $q \geq 0$, see for instance Bertoin et al. (2008); Bertoin and Yor (2005); Pardo et al. (2013). For a Lévy process $\xi$ satisfying one of these assumptions, $I_{\infty}(\xi)$ or $I_{\mathbf{e}_{q}}(\xi)$ is finite almost surely with an absolute continuous density. According to Theorem 3.9 in Bertoin et al. (2008), there exists a density for $I_{\infty}(\xi)$, here denoted by $h$. In the case when $q>0$, the existence of the density of $I_{\mathbf{e}_{q}}(\xi)$ appears in Pardo et al. (2013). Most of the known results on $I_{\infty}(\xi)$ and $I_{\mathbf{e}_{q}}(\xi)$ are related to the knowledge of their densities or the behaviour of their tail distributions.

From Theorem 2.2. in Kuznetsov et al. (2012), under the assumption that $\mathbb{E}\left[\left|\xi_{1}\right|\right]<\infty$, the density $h$ is completely determined by the following integral equation: for $v>0$,

$$
\begin{aligned}
\delta \int_{v}^{\infty} h(x) \mathrm{d} x+\frac{\rho^{2}}{2} v h(v) & +\int_{v}^{\infty} \overline{\bar{\Pi}}^{(-)}\left(\ln \frac{x}{v}\right) h(x) \mathrm{d} x \\
& +\int_{0}^{v} \overline{\bar{\Pi}}^{(+)}\left(\ln \frac{x}{v}\right) h(x) \mathrm{d} x+\int_{v}^{\infty} \frac{h(x)}{x} \mathrm{~d} x=0,
\end{aligned}
$$

where

$$
\overline{\bar{\Pi}}^{(+)}(x)=\int_{x}^{\infty} \int_{y}^{\infty} \Pi(\mathrm{d} z) \mathrm{d} y \quad \text { and } \quad \overline{\bar{\Pi}}^{(-)}(x)=\int_{x}^{\infty} \int_{-\infty}^{-y} \Pi(\mathrm{d} z) \mathrm{d} y .
$$


We refer to Bertoin et al. (2008); Bertoin and Yor (2005); Kuznetsov et al. (2012); Pardo et al. (2013), and the references therein, for more details about these facts.

In this paper, we are interested in the case when the Lévy process $\xi$ does not satisfy such conditions, in other words when $I_{t}(\xi)$ does not converge almost surely to a finite random variable, as $t$ goes to $\infty$. More precisely, one of our aims is to study the asymptotic behaviour of

$$
\mathbb{E}\left[F\left(I_{t}(\xi)\right)\right] \quad \text { as } \quad t \rightarrow \infty
$$

where $F$ is a function which, at infinity, is non-increasing and has polynomial decay, under some exponential moment conditions on $\xi$. We find five regimes that depend on the shape of the Laplace exponent $\psi$. These results will be applied in Section 2 for some particular cases such as

$$
F(x)=x^{-p}, \quad F(x)=1-e^{x^{-p}}, \quad F(x)=e^{-x}, \quad \text { or } \quad F(x)=\frac{a}{b+x},
$$

for $a, b, p, x>0$. Up to our knowledge, the case when the exponential functional of a Lévy process does not converge has only been studied in a few papers and not in its most general form, see for instance Bansaye et al. (2013); Böinghoff and Hutzenthaler (2012); Palau and Pardo (2016+). In all these papers, the main motivation comes from the study of some asymptotic properties of stochastic processes in random environment, that we briefly describe.

A recent manuscript authored by Li and Xu (2016), that appeared on Arxiv at the same time this manuscript was prepared, also studied the asymptotic behaviour of exponential functionals for Lévy processes. They obtained interesting results which are similar to the ones we present below using fluctuation theory for Lévy processes and the knowledge of Lévy processes conditioned to stay positive. Our approach is completely different and is based on a discretization of the exponential functional of Lévy processes and on the asymptotic behaviour of functionals of semi-direct products of random variables which was described by Guivarc'h and Liu (2001).

We would like to stress that Bansaye et al. (2013) already used a discretization technique to get the asymptotic behaviour of the survival probability for branchnig processes with catastrophes. However the discretization used in Bansaye et al. (2013) is different and only covers the case of compound Poisson processes.

Branching processes in random environment (BPREs) have been introduced and first studied by Smith and Wilkinson. This type of process has attracted considerable interest in the last decade (see for instance Afanasyev et al., 2012, 2005; Bansaye and Berestycki, 2009; Böinghoff et al., 2010 and the references therein). One of the reason is that BPREs are more realistic models than classical branching processes. And, from the mathematical point of view, they have interesting features such as a phase transition in the subcritical regime. Scaling limits for BPREs have been studied by Kurtz (1978) in the continuous case and more recently by Bansaye and Simatos (2015) in a more general setting.

Continuous state branching processes (CB-processes for short) in random environment, the continuous analogue in time and state space of BPREs, can be defined as a strong solution of a particular stochastic differential equation. They have been studied recently by several authors in different settings. Böinghoff and 
Hutzenthaler (2012) and Bansaye et al. (2013) studied extinction rates for branching diffusions in a Brownian environment and branching processes in a random environment driven by a Lévy process with bounded variations, respectively. Motivated by these works, Palau and Pardo $(2016+)$ studied the long-term behaviour (extinction, explosion, conditioned version) of branching processes in a Brownian random environment. In all these manuscripts, the existence of such processes is proved via a stochastic differential equation and it is observed that the speed of extinction is related to an exponential functional of a Lévy process which is associated to the random environment. Moreover, and similarly to the case of BPREs, a phase transition in the subcritical regime appears. Recently, branching processes in a general Lévy random environment were introduced by Palau and Pardo (2015).

Exponential functionals occur naturally in the study of some models of diffusions in random environment, which we now describe informally. Associated with a stochastic process $V=(V(x), x \in \mathbb{R})$ such that $V(0)=0$, a diffusion $X_{V}=$ $\left(X_{V}(t), t \geq 0\right)$ in the random potential $V$ is, loosely speaking, a solution to the stochastic differential equation

$$
\mathrm{d} X_{V}(t)=\mathrm{d} \beta_{t}-\frac{1}{2} V^{\prime}\left(X_{V}(t)\right) \mathrm{d} t, \quad X_{V}(0)=0
$$

where $\left(\beta_{t}, t \geq 0\right)$ is a standard Brownian motion independent of $V$. More rigorously, the process $X_{V}$ should be considered as a diffusion whose conditional generator, given $V$, is:

$$
\frac{1}{2} \exp (V(x)) \frac{\mathrm{d}}{\mathrm{d} x}\left(e^{-V(x)} \frac{\mathrm{d}}{\mathrm{d} x}\right) .
$$

Observe that from Feller's construction of such diffusions, the potential $V$ does not need to be differentiable. Kawazu and Tanaka (1993) studied the asymptotic behaviour of the tail of the distribution of the maximum of a diffusion in a drifted Brownian potential. Carmona et al. (1997) considered the case when the potential is a Lévy process whose jump structure is of bounded variation. More precisely, they studied the following question: How fast does $\mathbb{P}\left(\max _{t \geq 0} X_{V}(t)>x\right)$ decay as $x$ go to infinity? From these works, we know that

$$
\mathbb{P}\left(\max _{t \geq 0} X_{V}(t)>x\right)=\mathbb{E}\left[\frac{\tilde{I}}{\tilde{I}+I_{x}(-V)}\right]
$$

where

$$
\tilde{I}=\int_{-\infty}^{0} e^{V(t)} \mathrm{d} t \quad \text { and } \quad I_{x}(-V)=\int_{0}^{x} e^{V(t)} \mathrm{d} t
$$

are independent. As a consequence, exponential functionals play an essential role in this domain.

Let us now state our main results. Assume that

$$
\theta^{+}=\sup \{\lambda>0: \psi(\lambda)<\infty\}
$$

exists and is positive. In other words, the Laplace exponent of the Lévy process $\xi$ can be defined on $\left[0, \theta^{+}\right)$, see for instance Lemma 26.4 in Sato (2013). Besides, $\psi$ satisfies

$$
\psi(\lambda)=\log \mathbb{E}\left[e^{\lambda \xi_{1}}\right], \quad \lambda \in\left[0, \theta^{+}\right)
$$


From Theorem 25.3 in Sato (2013), $\psi(\lambda)<\infty$ is equivalent to

$$
\int_{\{|x|>1\}} e^{\lambda x} \Pi(\mathrm{d} x)<\infty .
$$

Moreover $\psi$ belongs to $C^{\infty}\left(\left[0, \theta^{+}\right)\right)$with $\psi(0)=0, \psi^{\prime}(0+) \in[-\infty, \infty)$ and $\psi^{\prime \prime}(\lambda)>$ 0 , for $\lambda \in\left(0, \theta^{+}\right)$(see Lemma 26.4 in Sato, 2013). Hence, the Laplace exponent $\psi$ is a convex function on $\left[0, \theta^{+}\right)$implying that either it is always positive or it may take negative values. In the latter scenario, $\psi$ may have at most one global minimum on $\left(0, \theta^{+}\right)$. Whenever such a global minimum exists, we denote by $\tau$ the position where it is reached. As we will see below, the shape of $\psi$ is essential to determine the asymptotic behaviour of $\mathbb{E}\left[I_{t}(\xi)^{-p}\right]$, for $0<p<\theta^{+}$.

Let us introduce the exponential change of measure known as the Esscher transform. According to Theorem 3.9 in Kyprianou (2006), for any $\lambda$ such that (1.3) is satisfied, we can perform the following change of measure

$$
\left.\frac{\mathrm{d} \mathbb{P}^{(\lambda)}}{\mathrm{d} \mathbb{P}}\right|_{\mathcal{F}_{t}}=e^{\lambda \xi_{t}-\psi(\lambda) t}, \quad t \geq 0
$$

where $\left(\mathcal{F}_{t}\right)_{t \geq 0}$ is the natural filtration generated by $\xi$ which is naturally completed. Moreover, under $\mathbb{P}^{(\lambda)}$ the process $\xi$ is still a Lévy process with Laplace exponent given by

$$
\psi_{\lambda}(z)=\psi(\lambda+z)-\psi(\lambda), \quad z \in \mathbb{R}
$$

Theorem 1.1. Assume that $0<p<\theta^{+}$.

i) If $\psi^{\prime}(0+)>0$, then

$$
\lim _{t \rightarrow \infty} \mathbb{E}\left[I_{t}(\xi)^{-p}\right]=\mathbb{E}\left[I_{\infty}(\xi)^{-p}\right]>0
$$

ii) If $\psi^{\prime}(0+)=0$ and $\psi^{\prime \prime}(0+)<\infty$, then there exists a positive constant $c_{1}$ such that

$$
\lim _{t \rightarrow \infty} \sqrt{t} \mathbb{E}\left[I_{t}(\xi)^{-p}\right]=c_{1}
$$

iii) Assume that $\psi^{\prime}(0+)<0$

a) if $\psi^{\prime}(p)<0$, then

$$
\lim _{t \rightarrow \infty} e^{-t \psi(p)} \mathbb{E}\left[I_{t}(\xi)^{-p}\right]=\mathbb{E}^{(p)}\left[I_{\infty}(-\xi)^{-p}\right]>0 .
$$

b) if $\psi^{\prime}(p)=0$ and $\psi^{\prime \prime}(p)<\infty$, then there exists a positive constant $c_{2}$ such that

$$
\lim _{t \rightarrow \infty} \sqrt{t} e^{-t \psi(p)} \mathbb{E}\left[I_{t}(\xi)^{-p}\right]=c_{2} .
$$

c) $\psi^{\prime}(p)>0$ and $\psi^{\prime \prime}(\tau)<\infty$ then

$$
\mathbb{E}\left[I_{t}(\xi)^{-p}\right]=o\left(t^{-1 / 2} e^{t \psi(\tau)}\right), \quad \text { as } \quad t \rightarrow \infty .
$$

Moreover if we also assume that $\xi$ is non-arithmetic (or non-lattice) then

$$
\mathbb{E}\left[I_{t}(\xi)^{-p}\right]=O\left(t^{-3 / 2} e^{t \psi(\tau)}\right), \quad \text { as } \quad t \rightarrow \infty .
$$

It is important to note that for any $q>0$ satisfying (1.3) for $\lambda=q$, we necessarily have that $\mathbb{E}\left[I_{t}(\xi)^{-q}\right]$ is finite for all $t>0$. Indeed, since $\left(e^{q \xi_{t}-t \psi(q)}, t \geq 0\right)$ is a positive martingale, we deduce from $L_{1}$-Doob's inequality (see for instance Acciaio 
et al., 2013) and the Esscher transform (1.4), that the following series of inequalities hold: for $t \leq 1$,

$$
\begin{aligned}
\mathbb{E}\left[I_{t}(\xi)^{-q}\right] & \leq t^{-q} \mathbb{E}\left[\sup _{0 \leq u \leq 1} e^{q \xi_{u}}\right] \leq t^{-q} e^{\psi(q) \vee 0} \mathbb{E}\left[\sup _{0 \leq u \leq 1} e^{q \xi_{u}-u \psi(q)}\right] \\
& \leq t^{-q} \frac{e^{1+\psi(q) \vee 0}}{e-1}\left(1+\mathbb{E}^{(q)}\left[q \xi_{1}-\psi(q)\right]\right) \\
& =t^{-q} \frac{e^{1+\psi(q) \vee 0}}{e-1}\left[1+q \psi^{\prime}(q)-\psi(q)\right],
\end{aligned}
$$

which is finite. The finiteness for $t>1$ follows from the fact that $I_{t}(\xi)$ is nondecreasing.

We are now interested in extending the above result for a class of functions which have polynomial decay and are non-increasing at $\infty$. As we will see below such extension is not straightforward and need more conditions on the exponential moments of the Lévy process $\xi$.

For simplicity, we write

$$
\mathcal{E}_{F}(t):=\mathbb{E}\left[F\left(I_{t}(\xi)\right)\right]
$$

where $F$ belongs to a particular class of continuous functions on $\mathbb{R}_{+}$that we will introduce below. Let $\mathbf{k}$ be a positive constant. We will consider functions $F$ satisfying one of the following conditions: There exists $x_{0} \geq 0$ such that $F(x)$ is non-increasing for $x \geq x_{0}$, and

(A1) $F$ satisfies

$$
F(x)=\mathbf{k}(x+1)^{-p}\left[1+(1+x)^{-\varsigma} h(x)\right], \quad \text { for all } x>0,
$$

where $0<p \leq \tau, \varsigma \geq 1$ and $h$ is a Lipschitz function which is bounded.

(A2) $F$ is an Hölder function with index $\alpha>0$ satisfying

$$
F(x) \leq \mathbf{k}(x+1)^{-p}, \quad \text { for all } x>0,
$$

with $p>\tau$.

Theorem 1.2. Assume that $0<p<\theta^{+}$. We have the following five regimes for the asymptotic behaviour of $\mathcal{E}_{F}(t)$ for large $t$.

i) If $\psi^{\prime}(0+)>0$ and $F$ is a positive and continuous function which is bounded, then

$$
\lim _{t \rightarrow \infty} \mathcal{E}_{F}(t)=\mathcal{E}_{F}(\infty)
$$

ii) If $\psi^{\prime}(0+)=0, F$ satisfies (A2) and $\theta^{-}:=\inf \{\lambda<0: \psi(\lambda)<\infty\}$ exists and is strictly negative, then there exists a positive constant $C_{1}$ such that

$$
\lim _{t \rightarrow \infty} \sqrt{t} \mathcal{E}_{F}(t)=C_{1} \text {. }
$$

iii) Suppose that $\psi^{\prime}(0+)<0$ :

a) If $F$ satisfies (A1) and $\psi^{\prime}(p)<0$, then, $\lim _{t \rightarrow \infty} e^{-t \psi(p)} \mathcal{E}_{F}(t)=\lim _{t \rightarrow \infty} e^{-t \psi(p)} \mathbf{k} \mathbb{E}\left[I_{t}(\xi)^{-p}\right]=\mathbf{k} \mathbb{E}^{(p)}\left[I_{\infty}(-\xi)^{-p}\right]$.

b) If F satisfies (A1), $\psi^{\prime}(p)=0$ and $\psi^{\prime \prime}(p)<\infty$, then, $\lim _{t \rightarrow \infty} \sqrt{t} e^{-t \psi(p)} \mathcal{E}_{F}(t)=\lim _{t \rightarrow \infty} \sqrt{t} e^{-t \psi(p)} \mathbf{k} \mathbb{E}\left[I_{t}(\xi)^{-p}\right]=\mathbf{k} c_{2}$, where $c_{2}$ has been defined in point iii) b) of Theorem 1.1. 
c) If $F$ satisfies (A2), $\psi^{\prime}(p)>0$ and $\tau+p<\theta^{+}$then there exists a positive constant $C_{3}$ such that

$$
\lim _{t \rightarrow \infty} t^{3 / 2} e^{-t \psi(\tau)} \mathcal{E}_{F}(t)=C_{3}
$$

The remainder of the paper is structured as follows. In Section 2, we apply our results to self-similar CB-processes, a population model with competition and diffusions whose dynamics are perturbed by a Lévy random environment. In particular, we study the asymptotic behaviour of the probability of extinction and explosion for some classes of self-similar CB-processes in a Lévy random environment. For the population model with competition, we describe the asymptotic behaviour of its mean. For the diffusion in a Lévy random environment, we provide the asymptotic behaviour of the tail probability of its global maximum. Finally, Section 3 is devoted to the proofs of Theorems 1.1 and 1.2 .

\section{Applications}

2.1. Self-similar CB-processes in a Lévy random environment. A $[0, \infty]$-valued strong Markov process $Y=\left(Y_{t}, t \geq 0\right)$ with probabilities $\left(\mathbb{P}_{x}, x \geq 0\right)$ is called a CB-process if it has càdlàg paths and its law satisfies the branching property; i.e. for any $x, y \geq 0, \mathbb{P}_{x+y}$ is equal in law to the convolution of $\mathbb{P}_{x}$ and $\mathbb{P}_{y}$. The law of $Y$ is completely characterized by its Laplace transform

$$
\mathbb{E}_{x}\left[e^{-\lambda Y_{t}}\right]=e^{-x u_{t}(\lambda)}, \quad \forall x>0, t \geq 0
$$

where $u$ is a differentiable function in $t$ satisfying

$$
\frac{\partial u_{t}(\lambda)}{\partial t}=-\Psi\left(u_{t}(\lambda)\right), \quad u_{0}(\lambda)=\lambda
$$

The function $\Psi$ is known as the branching mechanism of $Y$ and satisfies the celebrated Lévy-Khintchine formula

$$
\Psi(\lambda)=-a \lambda+\gamma^{2} \lambda^{2}+\int_{(0, \infty)}\left(e^{-\lambda x}-1+\lambda x \mathbf{1}_{\{x<1\}}\right) \mu(\mathrm{d} x), \quad \lambda \geq 0,
$$

where $a \in \mathbb{R}, \gamma \geq 0$ and $\mu$ is a measure concentrated on $(0, \infty)$ such that $\int_{(0, \infty)}(1 \wedge$ $\left.x^{2}\right) \mu(\mathrm{d} x)<\infty$.

Here we are interested in the case where the branching mechanism is stable, that is to say

$$
\Psi(\lambda)=c_{\beta} \lambda^{\beta+1}, \quad \lambda \in \mathbb{R},
$$

for some $\beta \in(-1,0) \cup(0,1]$ and $c_{\beta}$ is such that $\beta c_{\beta}>0$. We call its associated CB-process a self-similar CB-process. Under this assumption, the process $Y$ can also be defined as the unique non-negative strong solution of the following SDE (see for instance Fu and Li, 2010)

$$
Y_{t}=Y_{0}+\mathbf{1}_{\{\beta=1\}} \int_{0}^{t} \sqrt{2 c_{\beta} Y_{s}} \mathrm{~d} B_{s}+\mathbf{1}_{\{\beta \neq 1\}} \int_{0}^{t} \int_{0}^{\infty} \int_{0}^{Y_{s-}} z \widehat{N}(\mathrm{~d} s, \mathrm{~d} z, \mathrm{~d} u),
$$

where $B=\left(B_{t}, t \geq 0\right)$ is a standard Brownian motion, $N$ is a Poisson random measure independent of $B$ with intensity

$$
\frac{c_{\beta} \beta(\beta+1)}{\Gamma(1-\beta)} \frac{1}{z^{2+\beta}} \mathrm{d} s \mathrm{~d} z \mathrm{~d} u
$$


$\widetilde{N}$ is its compensated version and

$$
\widehat{N}(\mathrm{~d} s, \mathrm{~d} z, \mathrm{~d} u)= \begin{cases}N(\mathrm{~d} s, \mathrm{~d} z, \mathrm{~d} u) & \text { if } \beta \in(-1,0), \\ \widetilde{N}(\mathrm{~d} s, \mathrm{~d} z, \mathrm{~d} u) & \text { if } \beta \in(0,1)\end{cases}
$$

According to Palau and Pardo (2015), we can define a self-similar branching process whose dynamics are affected by a Lévy random environment (SSBLRE) as the unique non-negative strong solution of the stochastic differential equation

$$
\begin{aligned}
Z_{t}= & Z_{0}+\mathbf{1}_{\{\beta=1\}} \int_{0}^{t} \sqrt{2 c_{\beta} Z_{s}} \mathrm{~d} B_{s} \\
& +\mathbf{1}_{\{\beta \neq 1\}} \int_{0}^{t} \int_{0}^{\infty} \int_{0}^{Z_{s-}} z \widehat{N}(\mathrm{~d} s, \mathrm{~d} z, \mathrm{~d} u)+\int_{0}^{t} Z_{s-} \mathrm{d} S_{s},
\end{aligned}
$$

where

$S_{t}=\alpha t+\sigma W_{t}+\int_{0}^{t} \int_{(-1,1)}\left(e^{v}-1\right) \tilde{N}^{(e)}(\mathrm{d} s, \mathrm{~d} v)+\int_{0}^{t} \int_{\mathbb{R} \backslash(-1,1)}\left(e^{v}-1\right) N^{(e)}(\mathrm{d} s, \mathrm{~d} v)$,

$\alpha \in \mathbb{R}, \sigma \geq 0, W=\left(W_{t}, t \geq 0\right)$ is a standard Brownian motion, $N^{(e)}$ is a Poisson random measure in $\mathbb{R}_{+} \times \mathbb{R}$ independent of $W$ with intensity $\mathrm{d} s \pi(\mathrm{d} y), \widetilde{N}^{(e)}$ represents its compensated version and $\pi$ is a $\sigma$-finite measure concentrated on $\mathbb{R} \backslash\{0\}$ such that

$$
\int_{\mathbb{R}}\left(1 \wedge v^{2}\right) \pi(\mathrm{d} v)<\infty
$$

In addition, $Z$ satisfies the strong Markov property and, conditioned on the environment, the branching property. In what follows, we introduce the auxiliary Lévy process

$$
K_{t}=\mathbf{d} t+\sigma W_{t}+\int_{0}^{t} \int_{(-1,1)} v \widetilde{N}^{(e)}(\mathrm{d} s, \mathrm{~d} v)+\int_{0}^{t} \int_{\mathbb{R} \backslash(-1,1)} v N^{(e)}(\mathrm{d} s, \mathrm{~d} v),
$$

where

$$
\mathbf{d}=\alpha-\frac{\sigma^{2}}{2}-\int_{(-1,1)}\left(e^{v}-1-v\right) \pi(\mathrm{d} v) .
$$

The Laplace transform of $Z_{t} e^{-K_{t}}$ can be computed explicitly and provides a closed formula for the probabilities of survival and non explosion of $Z$. Indeed according to Proposition 1 in Palau and Pardo (2015), if $\left(Z_{t}, t \geq 0\right)$ is a stable SSBLRE with index $\beta \in(-1,0) \cup(0,1]$, then for all $z, \lambda>0$ and $t \geq 0$,

$$
\mathbb{E}_{z}\left[\exp \left\{-\lambda Z_{t} e^{-K_{t}}\right\} \mid K\right]=\exp \left\{-z\left(\lambda^{-\beta}+\beta c_{\beta} \int_{0}^{t} e^{-\beta K_{u}} \mathrm{~d} u\right)^{-1 / \beta}\right\} .
$$

Here, we are interested in two events which are of immediate concern for the process $Z$, explosion and extinction. The event of explosion before time $t$, is given by $\left\{Z_{t}=\infty\right\}$, and the event $\left\{\exists t>0, Z_{t}=0\right\}$ is referred as extinction.

2.1.1. Speed of explosion of SSBLRE. Let us first study the event of explosion for self-similar CB-processes in a Lévy random environment. It is important to note that this event has only been studied in the case when the random environment 
is driven by a Brownian motion with drift, see Palau and Pardo $(2016+)$. From Equation (2.4) and letting $\lambda$ go to 0 , we deduce

$$
\mathbb{P}_{z}\left(Z_{t}<\infty \mid K\right)=\mathbf{1}_{\{\beta>0\}}+\mathbf{1}_{\{\beta<0\}} \exp \left\{-z\left(\beta c_{\beta} \int_{0}^{t} e^{-\beta K_{u}} \mathrm{~d} u\right)^{-1 / \beta}\right\} \quad \text { a.s. }
$$

Let us focus on the most interesting case, $\beta \in(-1,0)$. Recall that when there is no environment, a self-similar CB-process explodes at time $t$ with probability $1-\exp \left\{-z\left(\beta c_{\beta} t\right)^{-1 / \beta}\right\}$. Under the presence of a Lévy random environment three different regimes appear for the asymptotic behaviour of the non-explosion probability. We call these regimes subcritical-explosion, critical-explosion or supercriticalexplosion depending on whether this probability stays positive, converges to zero polynomially fast or converges to zero exponentially fast.

Before stating our result, let us introduce the Laplace transform of the Lévy process $K$ by

$$
\kappa(\theta)=\log \mathbb{E}\left[e^{\theta K_{1}}\right]
$$

whenever it exists (see discussion on page 1238).

We assume that $\theta_{K}^{-}<0<\theta_{K}^{+}$, where

$$
\theta_{K}^{-}:=\inf \{\lambda<0: \kappa(\lambda)<\infty\} \text { and } \theta_{K}^{+}:=\sup \{\lambda>0: \kappa(\lambda)<\infty\} .
$$

As a consequence, the Laplace exponent $\kappa$ of $K$ is well defined on the interval $\left(\theta_{K}^{-}, \theta_{K}^{+}\right)$. If $\mathbf{m}:=\kappa^{\prime}(0+)>0$ then $\kappa$ takes only positive values on $\left(0, \theta_{K}^{+}\right)$, and some negatives values on $\left(\theta_{K}^{-}, 0\right)$. In this case, we may assume that $\kappa$ has a global minimum on $\left(\theta_{K}^{-}, 0\right)$ and we denote by $\hat{\tau}$ for its position. As we see below, the asymptotic behaviour of the probability of explosion depends on the sign of $\mathbf{m}$.

Proposition 2.1. Let $\left(Z_{t}, t \geq 0\right)$ be the SSBLRE with index $\beta \in(-1,0)$ defined by the $S D E$ (2.1) with $Z_{0}=z>0$, and recall the definition of the random environment $K$ in (2.3). Assume that $0<\theta_{K}^{+}$.

i) Subcritical-explosion. If $\mathbf{m}<0$, then, for every $z>0$

$$
\lim _{t \rightarrow \infty} \mathbb{P}_{z}\left(Z_{t}<\infty\right)=\mathbb{E}\left[\exp \left\{-z\left(\beta c_{\beta} \int_{0}^{\infty} e^{-\beta K_{u}} \mathrm{~d} u\right)^{-1 / \beta}\right\}\right]>0 .
$$

ii) Critical-explosion. If $\mathbf{m}=0$, then for every $z>0$ there exists $c_{1}(z)>0$ such that

$$
\lim _{t \rightarrow \infty} \sqrt{t} \mathbb{P}_{z}\left(Z_{t}<\infty\right)=c_{1}(z)
$$

iii) Supercritical-explosion. If $\mathbf{m}>0$, then for every $z>0$ there exists $c_{2}(z)>$ 0 such that

$$
\lim _{t \rightarrow \infty} t^{\frac{3}{2}} e^{t \kappa(\hat{\tau})} \mathbb{P}_{z}\left(Z_{t}<\infty\right)=c_{2}(z) .
$$

Proof: Observe that the function

$$
F: x \in \mathbb{R}_{+} \mapsto \exp \left(-z\left(\beta c_{\beta} x\right)^{-1 / \beta}\right)
$$

is non-increasing, continuous, bounded, and satisfies Assumption (A2) for every positive $p$. We also note that the processes $\left(\beta K_{t}, t \geq 0\right)$ and $\left(K_{t}, t \geq 0\right)$ have oposite signs implying

$$
\hat{\kappa}(\theta):=\log \mathbb{E}\left[e^{\beta \theta K_{1}}\right]=\kappa(\beta \theta),
$$


is well defined for $\theta \in\left(\theta_{K}^{+} / \beta, \theta_{K}^{-} / \beta\right)$. Hence Proposition 2.1 is a direct application of Theorem 1.2 points i), ii) and iii) c) with $\left(\xi_{t}, t \geq 0\right)=\left(\beta K_{t}, t \geq 0\right)$.

2.1.2. Speed of extinction of SSBLRE. Let us now focus on the survival probability. Throughout this section, we assume that $\beta \in(0,1]$. From Equation (2.4) and taking $\lambda$ go to $\infty$, we get

$$
\mathbb{P}_{z}\left(Z_{t}>0 \mid K\right)=1-\exp \left\{-z\left(\beta c_{\beta} \int_{0}^{t} e^{-\beta K_{u}} \mathrm{~d} u\right)^{-1 / \beta}\right\} \quad \text { a.s. }
$$

Similarly as for the explosion probability, the asymptotic behaviour of the probability depends on the sign of $\mathbf{m}=\kappa^{\prime}(0+)$. But in contrast with the explosion probability, five regimes appear, and a second parameter to take into account is the sign of

$$
\mathbf{m}_{1}=\kappa^{\prime}(1) \text {. }
$$

We recall that the Laplace exponent $\kappa$ of $K$ is well defined on the interval $\left(\theta_{K}^{-}, \theta_{K}^{+}\right)$ with $\theta_{K}^{-}<0<\theta_{K}^{+}$. In the case when $\mathbf{m}<0$, we may also assume that $\kappa$ has a global minimum on $\left(0, \theta_{K}^{+}\right)$and we denote by $\tau$ for its position.

Proposition 2.2. Let $\left(Z_{t}, t \geq 0\right)$ be a SSBLRE with index $\beta \in(0,1]$ defined by the $S D E$ (2.1) with $Z_{0}=z>0$, and recall the definition of the random environment $K$ in (2.3). Assume that $1<\theta_{K}^{+}$.

i) Supercritical case. If $\mathbf{m}>0$, then for every $z>0$

$$
\lim _{t \rightarrow \infty} \mathbb{P}_{z}\left(Z_{t}>0\right)=\mathbb{E}\left[1-\exp \left\{-z\left(\beta c_{\beta} \int_{0}^{\infty} e^{-\beta K_{u}} \mathrm{~d} u\right)^{-1 / \beta}\right\}\right]>0 .
$$

ii) Critical case. If $\mathbf{m}=0$, then for every $z>0$, there exists $c_{1}(z)>0$ such that

$$
\lim _{t \rightarrow \infty} \sqrt{t} \mathbb{P}_{z}\left(Z_{t}>0\right)=c_{1}(z) .
$$

iii) Subcritical case. Assume that $\mathbf{m}<0$, then

a) (Strongly subcritical regime). If $\mathbf{m}_{1}<0$, then there exists $c_{2}>0$ such that for every $z>0$,

$$
\lim _{t \rightarrow \infty} e^{-t \kappa(1)} \mathbb{P}_{z}\left(Z_{t}>0\right)=c_{2} z
$$

b) (Intermediate subcritical regime) If $\mathbf{m}_{1}=0$, then there exists $c_{3}>0$ such that for every $z>0$,

$$
\lim _{t \rightarrow \infty} \sqrt{t} e^{-t \kappa(1)} \mathbb{P}_{z}\left(Z_{t}>0\right)=c_{3} z,
$$

c) (Weakly subcritical regime) If $\mathbf{m}_{1}>0$, then for every $z>0$, there exists $c_{4}(z)>0$ such that

$$
\lim _{t \rightarrow \infty} t^{3 / 2} e^{-t \kappa(\tau)} \mathbb{P}_{z}\left(Z_{t}>0\right)=c_{4}(z) .
$$

Proof: This is a direct application of Theorem 1.2, with $\left(\xi_{t}, t \geq 0\right)=\left(\beta K_{t}, t \geq 0\right)$ and $F(x)=1-\exp \left(-z\left(\beta c_{\beta} x\right)^{-1 / \beta}\right)$.

In the strongly and intermediate subcritical cases $a$ ) and $b), \mathbb{E}\left[Z_{t}\right]$ provides the exponential decay factor of the survival probability which is given by $\kappa(1)$, and the probability of non-extinction is proportional to the initial state $z$ of the population. In the weakly subcritical case $c$ ), the survival probability decays exponentially with 
rate $\kappa(\tau)$, which is strictly smaller than $\kappa(1)$, and $c_{4}$ may not be proportional to $z$ (it is also the case for $c_{1}$ ). We refer to Bansaye (2009) for a result in this vein for discrete branching processes in random environment.

More generally, the results stated above can be compared to the results which appear in the literature of discrete (time and space) branching processes in random environment, see e.g. Afanasyev et al. (2005); Geiger et al. (2003); Guivarc'h and Liu (2001). In the continuous framework, such results have been established in Böinghoff and Hutzenthaler (2012) for the Feller diffusion case (i.e. $\beta=1$ ) in a Brownian environment, in Palau and Pardo (2016+) for a general CB-process in a Brownian environment, and in Bansaye et al. (2013) for stable CB-process $(\beta \in(0,1])$ subject to random catastrophes killing a fraction of the population.

2.2. Population model with competition in a Lévy random environment. We now study an extension of the competition model introduced in Evans et al. (2014) and studied by Palau and Pardo (2015). Following Palau and Pardo (2015), we define a logistic process with competition in a Lévy random environment, $\left(Z_{t}, t \geq 0\right)$, as the unique strong solution of the SDE

$$
Z_{t}=Z_{0}+\int_{0}^{t} Z_{s}\left(r-k Z_{s}\right) \mathrm{d} s+\int_{0}^{t} Z_{s-} \mathrm{d} S_{s}
$$

where $r>0$ is the drift, $k>0$ is the competition, and the environment $S$ is given by the Lévy process defined in (2.2). Moreover, the process $Z$ satisfies the Markov property and we have

$$
Z_{t}=Z_{0} e^{K_{t}}\left(1+k Z_{0} \int_{0}^{t} e^{K_{s}} \mathrm{~d} s\right)^{-1}, \quad t \geq 0,
$$

where $K$ is the Lévy process defined in (2.3).

The following result studies the asymptotic behaviour of $\mathbb{E}_{z}\left[Z_{t}\right]$, where $\mathbb{P}_{z}$ denotes the law of $Z$ starting from $z$. Before stating our result, let us recall the definition of the Laplace transform $\kappa$ of $K$ in (2.6) and that $\tau$ is the position of its global minimum, $\mathbf{m}=\kappa^{\prime}(0)$ and $\mathbf{m}_{1}=\kappa^{\prime}(1)$.

Proposition 2.3. Assume that $1<\theta_{K}^{+}$. For $z>0$, we have the following five regimes for the asymptotic behaviour of $\mathbb{E}_{z}\left[Z_{t}\right]$.

i) If $\mathbf{m}>0$, then for every $z>0$

$$
\lim _{t \rightarrow \infty} \mathbb{E}_{z}\left[Z_{t}\right]=\frac{1}{k} \mathbb{E}\left[\frac{1}{I_{\infty}(K)}\right]>0 .
$$

ii) If $\mathbf{m}=0$, then

$$
\mathbb{E}_{z}\left[Z_{t}\right]=O\left(t^{-1 / 2}\right)
$$

iii) Suppose that $\mathbf{m}<0$ :

a) If $\mathbf{m}_{1}<0$, then,

$$
\lim _{t \rightarrow \infty} e^{-t \kappa(1)} \mathbb{E}_{z}\left[Z_{t}\right]=\mathbb{E}^{(1)}\left[\frac{z}{1+z k I_{\infty}(-K)}\right]>0,
$$

where $\mathbb{E}^{(1)}$ denotes the Esscher transform (1.4) of $K$ with $\lambda=1$.

b) If $\mathbf{m}_{1}=0$, then there exists a positive constant $c(z, k)$ that depends on $z$ and $k$ such that

$$
\lim _{t \rightarrow \infty} \sqrt{t} e^{-t \kappa(1)} \mathbb{E}_{z}\left[Z_{t}\right]=c(z, k) .
$$


c) If $\mathbf{m}_{1}>0$ and $\tau+1<\theta^{+}$then there exists a positive constant $c_{1}(z, k)$ that depends on $z$ and $k$ such that

$$
\lim _{t \rightarrow \infty} t^{3 / 2} e^{-t \kappa(\tau)} \mathbb{E}_{z}\left[Z_{t}\right]=c_{1}(z, k) .
$$

Proof: We first recall from Lemma II.2 in Bertoin (1996) that the time reversed process $\left(K_{t}-K_{(t-s)^{-}}, 0 \leq s \leq t\right)$ has the same law as $\left(K_{s}, 0 \leq s \leq t\right)$. Then

$$
e^{-K_{t}} I_{t}(-K)=e^{-K_{t}} \int_{0}^{t} e^{K_{t-s}} \mathrm{~d} s=\int_{0}^{t} e^{-\left(K_{t}-K_{t-s}\right)} \mathrm{d} s \stackrel{(d)}{=} \int_{0}^{t} e^{-K_{s}} \mathrm{~d} s=I_{t}(K),
$$

and

$$
\left(e^{-K_{t}}, e^{-K_{t}} I_{t}(-K)\right) \stackrel{(d)}{=}\left(e^{-K_{t}}, I_{t}(K)\right),
$$

where $\stackrel{(d)}{=}$ stands for equality in law. The above implies that

$$
\mathbb{E}_{z}\left[Z_{t}\right]=z \mathbb{E}\left[\left(e^{-K_{t}}+k z e^{-K_{t}} \int_{0}^{t} e^{K_{s}} \mathrm{~d} s\right)^{-1}\right]=z \mathbb{E}\left[\left(e^{-K_{t}}+k z I_{t}(K)\right)^{-1}\right] .
$$

Let us now prove part i). Assume that $\mathbf{m}>0$, then $K$ drifts to $\infty$ and $e^{-K_{t}}$ converges to 0 as $t$ goes to $\infty$. By Theorem 1 in Bertoin and Yor $(2005), I_{t}(K)$ converges a.s. to $I_{\infty}(K)$, a non-negative and finite limit as $t$ goes to $\infty$. We observe that the result follows from identity (2.8) and the Monotone Convergence Theorem.

Part ii) follows from the inequality

$$
\mathbb{E}_{z}\left[Z_{t}\right]=z \mathbb{E}\left[\left(e^{-K_{t}}+k z I_{t}(K)\right)^{-1}\right] \leq \mathbb{E}\left[\left(k I_{t}(K)\right)^{-1}\right]
$$

and Theorem 1.1 part (ii).

Finally, we prove part iii). Observe by applying the Esscher transform (1.4) with $\lambda=1$ that

$$
\mathbb{E}_{z}\left[Z_{t}\right]=z e^{\kappa(1) t} \mathbb{E}^{(1)}\left[\left(1+k z \int_{0}^{t} e^{K_{s}} \mathrm{~d} s\right)^{-1}\right]
$$

Part iii)-a) follows by observing that under the probability measure $\mathbb{P}^{(1)}$, the process $K$ is a Lévy process with mean $\mathbb{E}^{(1)}\left[K_{1}\right]=\kappa^{\prime}(1) \in(-\infty, 0)$. We then conclude as in the proof of part i) by showing that $\mathbb{E}^{(1)}\left[\left(1+k z I_{t}(-K)\right)^{-1}\right]$, converges to $\mathbb{E}^{(1)}\left[\left(1+k z I_{\infty}(-K)\right)^{-1}\right]$, as $t$ increases.

Finally parts iii)-b) and c) follow from a direct application of Theorem (1.2) parts iii)-b) and c), respectively, with the function $F: x \in \mathbb{R}_{+} \mapsto z(1+k z x)^{-1}$.

2.3. Diffusions in a Lévy random environment. Let $(V(x), x \in \mathbb{R})$ be a stochastic process defined on $\mathbb{R}$ such that $V(0)=0$. As presented in the introduction, a diffusion process $X=(X(t), t \geq 0)$ in a random potential $V$ is a diffusion whose conditional generator given $V$ is

$$
\frac{1}{2} e^{V(x)} \frac{\mathrm{d}}{\mathrm{d} x}\left(e^{-V(x)} \frac{\mathrm{d}}{\mathrm{d} x}\right)
$$

It is well known that $X$ may be constructed from a Brownian motion through suitable changes of scale and time, see Brox (1986).

The problem that we would like to study is the following: How fast does $\mathbb{P}\left(\max _{t \geq 0} X(t)>x\right)$ decay as $x$ go to infinity? In order to make our analysis more 
tractable, we consider $\left(\xi_{t}, t \geq 0\right)$ and $\left(\eta_{t}, t \geq 0\right)$ two independent Lévy processes, and we define

$$
V(x)=\left\{\begin{array}{lll}
-\xi_{x} & \text { if } \quad x \geq 0 \\
-\eta_{-x} & \text { if } \quad x \leq 0
\end{array}\right.
$$

We want to determine the asymptotic behaviour of

$$
\mathbb{P}\left(\max _{s \geq 0} X(s)>t\right)=\mathbb{E}\left[\frac{I_{\infty}(\eta)}{I_{\infty}(\eta)+I_{t}(\xi)}\right] .
$$

We assume that $\eta$ drifts to $\infty$, and recall the notations of Section 1 for the Laplace exponent $\psi$ of $\xi$.

Proposition 2.4. Assume that $1<\theta^{+}$.

i) If $\psi^{\prime}(0+)>0$, then

$$
\lim _{t \rightarrow \infty} \mathbb{P}\left(\max _{s \geq 0} X(s)>t\right)=\mathbb{E}\left[\frac{I_{\infty}(\eta)}{I_{\infty}(\eta)+I_{\infty}(\xi)}\right]>0 .
$$

ii) If $\psi^{\prime}(0+)=0$, then there exists a positive constant $C_{1}$ that depends on the law of $I_{\infty}(\eta)$ such that

$$
\lim _{t \rightarrow \infty} \sqrt{t} \mathbb{P}\left(\max _{s \geq 0} X(s)>t\right)=C_{1} .
$$

iii) Suppose that $\psi^{\prime}(0+)<0$ :

a) If $\psi^{\prime}(1)<0$, then there exists a positive constant $C_{2}$ that depends on the law of $I_{\infty}(\eta)$ such that,

$$
\lim _{t \rightarrow \infty} e^{-t \psi(1)} \mathbb{P}\left(\max _{s \geq 0} X(s)>t\right)=C_{2} .
$$

b) If $\psi^{\prime}(1)=0$ and $\psi^{\prime \prime}(1)<\infty$, then there exists a positive constant $C_{3}$ that depends on the law of $I_{\infty}(\eta)$ such that

$$
\lim _{t \rightarrow \infty} \sqrt{t} e^{-t \psi(1)} \mathbb{P}\left(\max _{s \geq 0} X(s)>t\right)=C_{3} .
$$

c) If $\psi^{\prime}(1)>0$, and $\tau+1<\theta^{+}$, then

$$
\lim _{t \rightarrow \infty} \mathbb{P}\left(\max _{s \geq 0} X(s)>t\right)=o\left(t^{-1 / 2} e^{-t \psi(\tau)}\right) .
$$

Moreover, if the process $\xi$ is non-arithmetic (or non-lattice) then there exists a positive constant $C_{4}$ that depends on the law of $I_{\infty}(\eta)$ such that

$$
\lim _{t \rightarrow \infty} t^{3 / 2} e^{-t \psi(\tau)} \mathbb{P}\left(\max _{s \geq 0} X(s)>t\right)=C_{4} .
$$

Furthermore, if there exists a positive $\varepsilon$ such that

$$
\mathbb{E}\left[I_{\infty}(\eta)^{1+\varepsilon}\right]<\infty,
$$

then

$$
C_{i}=c_{i} \mathbb{E}\left[I_{\infty}(\eta)\right], \quad i \in\{2,3\},
$$

where $\left(c_{i}, i \in\{2,3\}\right)$ do not depend on the law of $I_{\infty}(\eta)$. 
Proof: Since $\eta$ and $\xi$ are independent, we have

$\mathbb{P}\left(\max _{s \geq 0} X(s)>t\right)=\mathbb{E}\left[I_{\infty}(\eta) \mathbb{E}\left[\frac{1}{I_{\infty}(\eta)+I_{t}(\xi)} \mid \eta\right]\right]=\mathbb{E}\left[I_{\infty}(\eta) f\left(I_{\infty}(\eta), t\right)\right], t>0$,

where

$$
f(a, t)=\mathbb{E}\left[\left(a+I_{t}(\xi)\right)^{-1}\right], \quad a, t>0 .
$$

The result follows from an application of Theorems 1.1 and 1.2 with the function

$$
F: x \in \mathbb{R}_{+} \mapsto z(a+x)^{-1} .
$$

We only prove case ii), as the others are analogous. By Theorem 1.2 there exists $c_{1}(a)>0$ such that

$$
\lim _{t \rightarrow \infty} t^{1 / 2} f(a, t)=c_{1}(a) .
$$

On the other hand, by Theorem 1 , there exists $c_{1}$ such that

$$
\lim _{t \rightarrow \infty} t^{1 / 2} f(0, t)=c_{1} \text {. }
$$

Let us define $G_{t}(a)=a t^{1 / 2} f(a, t)$, and $G_{t}^{0}(a)=a t^{1 / 2} f(0, t)$. Observe that

$$
G_{t}(a) \leq G_{t}^{0}(a), \quad \text { for all } t, a \geq 0
$$

and

$$
\lim _{t \rightarrow \infty} \mathbb{E}\left[G_{t}^{0}\left(I_{\infty}(\eta)\right)\right]=c_{1} \mathbb{E}\left[I_{\infty}(\eta)\right] .
$$

Then, by the Dominated Convergence Theorem (see for instance Dudley, 2002 problem 12 p. 145),

$$
\lim _{t \rightarrow \infty} \sqrt{t} \mathbb{P}\left(\max _{s \geq 0} X(s)>t\right)=\lim _{t \rightarrow \infty} \mathbb{E}\left[G_{t}\left(I_{\infty}(\eta)\right)\right]=\mathbb{E}\left[I_{\infty}(\eta) c_{1}\left(I_{\infty}(\eta)\right)\right] .
$$

We complete the proof for the existence of the limits by observing that

$$
0<C_{1}=\mathbb{E}\left[I_{\infty}(\eta) c_{1}\left(I_{\infty}(\eta)\right)\right] \leq c_{1} \mathbb{E}\left[I_{\infty}(\eta)\right]<\infty .
$$

The last part of the proof consists in justifying the form of the constants $C_{2}$ and $C_{3}$ under the additional condition $\mathbb{E}\left[I_{\infty}(\eta)^{1+\varepsilon}\right]<\infty$ for a positive $\varepsilon$. For every $0 \leq \varepsilon \leq 1$, we have

$$
\begin{aligned}
\frac{I_{\infty}(\eta)}{I_{t}(\xi)}-\frac{I_{\infty}(\eta)}{I_{\infty}(\eta)+I_{t}(\xi)} & =\frac{I_{\infty}(\eta)}{I_{t}(\xi)} \frac{I_{\infty}(\eta)}{I_{\infty}(\eta)+I_{t}(\xi)} \\
& \leq \frac{I_{\infty}(\eta)}{I_{t}(\xi)}\left(\frac{I_{\infty}(\eta)}{I_{\infty}(\eta)+I_{t}(\xi)}\right)^{\varepsilon} \leq\left(\frac{I_{\infty}(\eta)}{I_{t}(\xi)}\right)^{1+\varepsilon} .
\end{aligned}
$$

Hence

$$
0 \leq \mathbb{E}\left[\frac{I_{\infty}(\eta)}{I_{t}(\xi)}-\frac{I_{\infty}(\eta)}{I_{\infty}(\eta)+I_{t}(\xi)}\right] \leq \mathbb{E}\left[\left(I_{\infty}(\eta)\right)^{1+\varepsilon}\right] \mathbb{E}\left[\frac{1}{\left(I_{t}(\xi)\right)^{1+\varepsilon}}\right]
$$

But from point iii)-c) of Theorem 1.1 and Equation (3.10) in the proof of Theorem 1.2, we know that in the cases iii)-a) and iii)-b),

$$
\mathbb{E}\left[I_{t}(\xi)^{-(1+\varepsilon)}\right]=o\left(\mathbb{E}\left[I_{t}(\xi)^{-1}\right]\right) \text {. }
$$

This ends the proof. 


\section{Proofs of Theorems 1.1 and 1.2.}

This section is dedicated to the proofs of the main results of the paper.

We first prove Theorem 1.1. The proof of part ii) is based on the following approximation technique. Let $\left(N_{t}^{(q)}, t \geq 0\right)$ be a Poisson process with intensity $q>0$, which is independent of the Lévy process $\xi$, and denote by $\left(\tau_{n}^{q}\right)_{n \geq 0}$ its sequence of jump times with the convention that $\tau_{0}^{q}=0$. For simplicity, we also introduce for $n \geq 0$,

$$
\xi_{t}^{(n)}=\xi_{\tau_{n}^{q}+t}-\xi_{\tau_{n}^{q}}, \quad t \geq 0 .
$$

For $n \geq 0$, we define the following random variables

$$
S_{n}^{(q)}:=\xi_{\tau_{n}^{q}}, \quad M_{n}^{(q)}:=\sup _{\tau_{n}^{q} \leq t<\tau_{n+1}^{q}} \xi_{t} \quad \text { and } \quad I_{n}^{(q)}:=\inf _{\tau_{n}^{q} \leq t<\tau_{n+1}^{q}} \xi_{t} .
$$

Observe that $\left(S_{n}^{(q)}, n \geq 0\right)$ is a random walk with step distribution given by $\xi_{\tau_{1}^{q}}$ and that $\tau_{1}^{q}$ is an exponential r.v. with parameter $q$ which is independent of $\xi$.

Similarly for the process $\xi^{(n)}$, we also introduce

$$
m_{n}^{(q)}:=\sup _{t<\tau_{n+1}^{q}-\tau_{n}^{q}} \xi_{t}^{(n)} \quad \text { and } \quad i_{n}^{(q)}:=\inf _{t<\tau_{n+1}^{q}-\tau_{n}^{q}} \xi_{t}^{(n)} .
$$

Lemma 3.1. Using the above notation we have

$$
M_{n}^{(q)}=S_{n}^{(+, q)}+m_{0}^{(q)}, \quad I_{n}^{(q)}=S_{n}^{(-, q)}+i_{0}^{(q)}, \quad n \geq 0
$$

where each of the processes $S^{(+, q)}=\left(S_{n}^{(+, q)}, n \geq 0\right)$ and $S^{(-, q)}=\left(S_{n}^{(-, q)}, n \geq 0\right)$ are random walks with the same distribution as $S^{(q)}$. Moreover $S^{(+, q)}$ and $m_{0}^{(q)}$ are independent, as are $S^{(-, q)}$ and $i_{0}^{(q)}$.

The proof of this lemma is based on the Wiener-Hopf factorisation (see Equations (4.3.3) and (4.3.4) in Doney, 2007). It follows from similar arguments as those used in the proof of Theorem IV.13 in Doney (2007), which considers the case when the exponential random variables are jump times of the process $\xi$ restricted to $\mathbb{R} \backslash[-\eta, \eta]$, for $\eta>0$. So, we omit its proof for the sake of brevity.

Recall that $\tau_{1}^{q}$ goes to 0 , in probability, as $q$ increases and that $\xi$ has càdlàg paths. Hence, there exists an increasing sequence $\left(q_{n}\right)_{n \geq 0}$ such that $q_{n} \rightarrow \infty$ and

$$
e^{\lambda i_{0}^{\left(q_{n}\right)}} \underset{n \rightarrow \infty}{\longrightarrow} 1, \quad \text { a.s. }
$$

We also recall the following form of the Wiener-Hopf factorisation, for $q>\psi(\lambda)$

$$
\frac{q}{q-\psi(\lambda)}=\mathbb{E}\left[e^{\lambda i_{0}^{(q)}}\right] \mathbb{E}\left[e^{\lambda m_{0}^{(q)}}\right]
$$

From the Dominated Convergence Theorem and identity (3.2), it follows that for $\varepsilon \in(0,1)$, there exists $N \in \mathbb{Z}_{+}$such that for all $n \geq N$

$$
1-\varepsilon \leq \mathbb{E}\left[e^{\lambda i_{0}^{\left(q_{n}\right)}}\right] \leq \mathbb{E}\left[e^{\lambda m_{0}^{\left(q_{n}\right)}}\right] \leq 1+\varepsilon
$$

Next, we introduce the compound Poisson process

$$
Y_{t}^{(q)}:=S_{N_{t}^{(q)}}^{(q)}, \quad t \geq 0
$$


whose Laplace exponent satisfies

$$
\psi^{(q)}(\lambda):=\log \mathbb{E}\left[e^{\lambda Y_{1}^{(q)}}\right]=\frac{q \psi(\lambda)}{q-\psi(\lambda)},
$$

which is well defined for $\lambda$ such that $q>\psi(\lambda)$. Similarly, we define

$\widetilde{I}_{t}^{(q)}=I_{N_{t}^{(q)}}^{(q)}, \quad \widetilde{M}_{t}^{(q)}=M_{N_{t}^{(q)}}^{(q)}, \quad Y_{t}^{(+, q)}=S_{N_{t}^{(q)}}^{(+, q)}, \quad$ and $\quad Y_{t}^{(-, q)}=S_{N_{t}^{(q)}}^{(-, q)}$.

We observe from the definitions of $\widetilde{M}^{(q)}$ and $\widetilde{I}^{(q)}$, and Lemma 3.1, that for all $t \geq 0$, the following inequalities are satisfied

$$
e^{-m_{0}^{(q)}} \int_{0}^{t} e^{-Y_{s}^{(+, q)}} \mathrm{d} s \leq \int_{0}^{t} e^{-\xi_{s}} \mathrm{~d} s \leq e^{-i_{0}^{(q)}} \int_{0}^{t} e^{-Y_{s}^{(-, q)}} \mathrm{d} s .
$$

We have now all the tools needed to prove Theorem 1.1.

Proof of Theorem 1.1: i) Assume that $\psi^{\prime}(0+)>0$. According to Theorem 1 in Bertoin and Yor (2005), $I_{t}(\xi)$ converges a.s. to $I_{\infty}(\xi)$, a non-negative and finite limit as $t$ goes to $\infty$. Then, we observe that the result follows from the Monotone Convergence Theorem.

We now prove part ii). In order to do so, we use the approximation and notation that we introduced at the beginning of this section. Let $\left(q_{n}\right)_{n \geq 1}$ be a sequence defined as in (3.1) and observe that for $n \geq 1$, we have $\psi^{\left(q_{n}\right)}(0)=0, \psi^{\prime\left(q_{n}\right)}(0+)=0$ and $\psi^{\prime \prime\left(q_{n}\right)}(0+)<\infty$. We also observe that the processes $Y^{\left(+, q_{n}\right)}$ and $Y^{\left(-, q_{n}\right)}$ have bounded variation paths.

We take $\ell \geq N$ and $0<\varepsilon<1$. Hence from Lemmas 13 and 14 in Bansaye et al. (2013), we observe that there exists a positive constant $c_{1}(\ell)$ such that

$$
(1-\varepsilon) c_{1}(\ell) t^{-1 / 2} \leq \mathbb{E}\left[\left(\int_{0}^{t} e^{-Y_{s}^{\left( \pm, q_{\ell}\right)}} \mathrm{d} s\right)^{-p}\right] \leq(1+\varepsilon) c_{1}(\ell) t^{-1 / 2},
$$

for $t$ sufficiently large. Therefore using (3.3) and (3.4) in the previous inequality, we obtain

$$
(1-\varepsilon)^{2} c_{1}(\ell) t^{-1 / 2} \leq \mathbb{E}\left[I_{t}(\xi)^{-p}\right] \leq(1+\varepsilon)^{2} c_{1}(\ell) t^{-1 / 2},
$$

for $t$ sufficiently large.

Next, we take $n, m \geq N$ and observe that the previous inequalities imply

$$
\left(\frac{1-\varepsilon}{1+\varepsilon}\right)^{2} c_{1}(n) \leq c_{1}(m) \leq\left(\frac{1+\varepsilon}{1-\varepsilon}\right)^{2} c_{1}(n), \quad \text { for all } n, m \geq N .
$$

Thus, we deduce that $\left(c_{1}(n)\right)_{n \geq 1}$ is a Cauchy sequence. Let us denote $c_{1}$ its limit which, by the previous inequalities is positive. Let $l \geq N$ such that

$$
(1-\varepsilon) c_{1} \leq c_{1}(l) \leq(1+\varepsilon) c_{1} .
$$

Using this inequality and (3.5), we observe

$$
(1-\varepsilon)^{3} c_{1} t^{-1 / 2} \leq \mathbb{E}\left[I_{t}(\xi)^{-p}\right] \leq(1+\varepsilon)^{3} c_{1} t^{-1 / 2},
$$

for $t$ sufficiently large. This completes the proof of part ii).

Now, we prove part iii)-a). Recalling (2.7) yields that

$$
I_{t}(\xi) \stackrel{(d)}{=} e^{-\xi_{t}} I_{t}(-\xi), \quad t \geq 0
$$


Hence using the Esscher transform (1.4), with $\lambda=p$, we have

$$
\mathbb{E}\left[I_{t}(\xi)^{-p}\right]=\mathbb{E}\left[e^{p \xi_{t}} I_{t}(-\xi)^{-p}\right]=e^{t \psi(p)} \mathbb{E}^{(p)}\left[I_{t}(-\xi)^{-p}\right], \quad t \geq 0
$$

The inequality (1.5) with $q=p$ and the previous identity imply that the decreasing function $t \mapsto \mathbb{E}^{(p)}\left[I_{t}(-\xi)^{-p}\right]$ is finite for all $t>0$. Recall that under the probability measure $\mathbb{P}^{(p)}$, the process $\xi$ is a Lévy process with mean $\mathbb{E}^{(p)}\left[\xi_{1}\right]=\psi^{\prime}(p) \in(-\infty, 0)$. Then, as in the proof of part i), $\mathbb{E}^{(p)}\left[I_{t}(-\xi)^{-p}\right]$ converges to $\mathbb{E}^{(p)}\left[I_{\infty}(-\xi)^{-p}\right]$, as $t$ increases.

Part iii)-b) follows from part ii) and the Esscher transform (1.4). More precisely, we apply the Esscher transform with $\lambda=p$ and observe that the Laplace transform of the process $\xi$ under the probability measure $\mathbb{P}^{(p)}$, satisfies $\psi_{p}^{\prime}(0+)=\psi^{\prime}(p)=0$ and $\psi_{p}^{\prime \prime}(0+)=\psi^{\prime \prime}(p)<\infty$. Therefore by applying part ii) and identity (3.7), we get the existence of a constant $c_{2}>0$ such that

$$
\mathbb{E}\left[I_{t}(\xi)^{-p}\right]=e^{t \psi(p)} \mathbb{E}^{(p)}\left[I_{t}(-\xi)^{-p}\right] \sim c_{2} t^{-1 / 2} e^{t \phi(p)}
$$

Finally we prove part iii)-c). Again from the Esscher transform with $\lambda=\tau$, we see

$$
\mathbb{E}\left[I_{t}(\xi)^{-p}\right]=e^{t \psi(\tau)} \mathbb{E}^{(\tau)}\left[e^{(p-\tau) \xi_{t}} I_{t}(-\xi)^{-p}\right], \quad t>0
$$

On the one hand, for $t>0$,

$$
\begin{aligned}
& E^{(\tau)}\left[e^{(p-\tau) \xi_{t}} I_{t}(-\xi)^{-p}\right] \\
& =\mathbb{E}^{(\tau)}\left[e^{(p-\tau)\left(\xi_{t}-\xi_{t / 2}\right)} \frac{\left(e^{\left.-\xi_{t / 2} I_{t / 2}(-\xi)+\int_{t / 2}^{t} e^{\xi_{u}-\xi_{t / 2}} \mathrm{~d} u\right)^{-(p-\tau)}}\right.}{\left(I_{t / 2}(-\xi)+e^{\xi_{t / 2}} \int_{t / 2}^{t} e^{\xi_{u}-\xi_{t / 2}} \mathrm{~d} u\right)^{\tau}}\right] \\
& \leq \mathbb{E}^{(\tau)}\left[e^{(p-\tau)\left(\xi_{t}-\xi_{t / 2}\right)} \frac{\left(\int_{0}^{t / 2} e^{\xi_{s+t / 2}-\xi_{t / 2}} \mathrm{~d} s\right)^{-(p-\tau)}}{I_{t / 2}(-\xi)^{\tau}}\right] \\
& =\mathbb{E}^{(\tau)}\left[e^{(p-\tau)\left(\xi_{t / 2}\right)} I_{t / 2}(-\xi)^{-(p-\tau)}\right] \mathbb{E}^{(\tau)}\left[I_{t / 2}(-\xi)^{-\tau}\right]
\end{aligned}
$$

where we have used in the last identity the fact that $\left(\xi_{u+t / 2}-\xi_{t / 2}, u \geq 0\right)$ is independent of $\left(\xi_{u}, 0 \leq u \leq t / 2\right)$ and with the same law as $\left(\xi_{u}, u \geq 0\right)$.

On the other hand, from (3.6) we deduce

$$
\mathbb{E}^{(\tau)}\left[e^{(p-\tau)\left(\xi_{t / 2}\right)} I_{t / 2}(-\xi)^{-(p-\tau)}\right]=\mathbb{E}^{(\tau)}\left[I_{t / 2}(\xi)^{-(p-\tau)}\right], \quad t>0
$$

Putting all the pieces together, we get

$$
\mathbb{E}^{(\tau)}\left[e^{(p-\tau) \xi_{t}} I_{t}(-\xi)^{-p}\right] \leq \mathbb{E}^{(\tau)}\left[I_{t / 2}(\xi)^{-(p-\tau)}\right] \mathbb{E}^{(\tau)}\left[I_{t / 2}(-\xi)^{-\tau}\right], \quad t>0
$$

implying

$$
\mathbb{E}\left[I_{t}(\xi)^{-p}\right] \leq e^{t \psi(\tau)} \mathbb{E}^{(\tau)}\left[I_{t / 2}(\xi)^{-(p-\tau)}\right] \mathbb{E}^{(\tau)}\left[I_{t / 2}(-\xi)^{-\tau}\right], \quad t>0 .
$$

Since $\psi^{\prime}(\tau)=0$, we have $\mathbb{E}^{(\tau)}\left[\xi_{1}\right]=0$ and the process $\xi$ oscillates under $\mathbb{P}^{(\tau)}$. Moreover since $\psi^{\prime \prime}(\tau)<\infty$, we deduce that $\psi_{\tau}^{\prime \prime}(0+)<\infty$. The latter condition implies from part ii) that there exists a constant $c_{1}(\tau)>0$ such that

$$
\mathbb{E}^{(\tau)}\left[I_{t}(\xi)^{-(p-\tau)}\right] \sim c_{1}(\tau) t^{-1 / 2}
$$


for $t$ sufficiently large. Since the process $\xi$ oscillates under $\mathbb{P}^{(\tau)}$, the dual $-\xi$ also oscillates. This implies that $I_{t}(-\xi)$ goes to $\infty$ and therefore $\mathbb{E}^{(\tau)}\left[I_{t}(-\xi)^{-(p-\tau)}\right]$ goes to 0 , as $t$ increases. In other words, we have

$$
\mathbb{E}\left[I_{t}(\xi)^{-p}\right]=o\left(t^{-1 / 2} e^{t \psi(\tau)}\right), \quad \text { as } \quad t \rightarrow \infty,
$$

as expected.

We now assume that $\xi$ is non-arithmetic, our arguments are similar to those used in Bansaye et al. (2013). We will prove

$$
\limsup _{t \rightarrow \infty} t^{3 / 2} e^{-t \psi(\tau)} \mathbb{E}\left[I_{t}(\xi)^{-p}\right]<\infty .
$$

In order to prove it, we take $t>0$ and observe

$$
I_{\lfloor t\rfloor}(\xi)=\sum_{k=0}^{\lfloor t\rfloor-1} e^{-\xi_{k}} \int_{0}^{1} e^{-\left(\xi_{k+u}-\xi_{k}\right)} \mathrm{d} u,
$$

where $\lfloor t\rfloor$ denotes the integer part of $t$. Therefore

$$
\mathbb{E}\left[I_{\lfloor t\rfloor}(\xi)^{-p}\right] \leq \mathbb{E}\left[\min _{k \leq\lfloor t\rfloor-1} e^{p \xi_{k}}\left(\int_{0}^{1} e^{-\left(\xi_{k+u}-\xi_{k}\right)} \mathrm{d} u\right)^{-p}\right] .
$$

Conditioning on the value when the minimum is attained, let say $k^{\prime}$, and observing that $e^{p \xi_{k^{\prime}}}$ is independent of $\left(\int_{0}^{1} e^{-\left(\xi_{k^{\prime}+u}-\xi_{k^{\prime}}\right)} \mathrm{d} u\right)^{-p}$ and the latter has the same law as $\left(\int_{0}^{1} e^{-\xi_{u}} \mathrm{~d} u\right)^{-p}$, we deduce

$$
\mathbb{E}\left[I_{\lfloor t\rfloor}(\xi)^{-p}\right] \leq \mathbb{E}\left[\min _{k \leq\lfloor t\rfloor-1} e^{p \xi_{k}}\right] \mathbb{E}\left[\left(\int_{0}^{1} e^{-\xi_{u}} \mathrm{~d} u\right)^{-p}\right] .
$$

Finally, by Lemma 7 in Hirano (1998), there exists a $C>0$ such that

$$
\mathbb{E}\left[\min _{k \leq\lfloor t\rfloor-1} e^{p \xi_{k}}\right] \sim C\lfloor t\rfloor^{-3 / 2} e^{\lfloor t\rfloor \psi(\tau)}, \quad \text { for } t \text { large. }
$$

The claim follows from the monotonicity of $\mathbb{E}\left[I_{\lfloor t\rfloor}(\xi)^{-p}\right]$ and the fact that $t \in$ $[\lfloor t\rfloor,\lfloor t\rfloor+1)$.

The idea of the proof of Theorem 1.2 is to study the asymptotic behaviour of $\mathcal{E}_{F}(n / q)$ for $q$ fixed and large $n$, and then to use the monotonicity of $F$ to deduce the asymptotic behaviour of $\mathcal{E}_{F}(t)$ when $t$ goes to infinity. In order to do so, we use a key result due to Guivarc'h and Liu that we state in the Appendix for the sake of completeness.

Let $q>0$ and define the sequence $q_{n}=n / q$, for $n \geq 0$. For $k \geq 0$, we also define

$$
\widetilde{\xi}_{u}^{(k)}=\xi_{q_{k}+u}-\xi_{q_{k}}, \quad \text { for } \quad u \geq 0
$$

and

$$
a_{k}=e^{-\widetilde{\xi}_{q_{(k+1)}}^{(k)}-q_{k}} \quad \text { and } \quad b_{k}=\int_{0}^{q_{(k+1)}-q_{k}} e^{-\widetilde{\xi}_{u}^{(k)}} \mathrm{d} u .
$$

Hence, $\left(a_{k}, b_{k}\right)$ is a $\mathbb{R}_{+}^{2}$-valued sequence of i.i.d. random variables. Observe that

$$
a_{0}=e^{-\xi_{\frac{1}{q}}} \quad \text { and } \quad \frac{b_{0}}{1-a_{0}}=\frac{I_{\frac{1}{q}}(\xi)}{1-e^{-\xi_{\frac{1}{q}}}},
$$


which are not constant a.s. as required by Theorem A.1. Moreover, we have

$$
\int_{q_{i}}^{q_{i+1}} e^{-\xi_{u}} \mathrm{~d} u=e^{-\xi_{q_{i}}} b_{i}=\prod_{k=0}^{i-1} a_{k} b_{i}=A_{i} b_{i}
$$

where $A_{k}$ is defined as in Theorem A.1. The latter identity implies

$$
I_{q_{n}}(\xi)=\sum_{i=0}^{n-1} \int_{q_{i}}^{q_{i+1}} e^{-\xi_{u}} \mathrm{~d} u=\sum_{i=0}^{n-1} A_{i} b_{i}:=B_{n}
$$

In other words, we have all the objects required to apply Theorem A.1.

Proof of Theorem 1.2: i) The proof uses similar arguments as those used in the proof of Theorem 1.1-i).

ii) We now assume that $\psi^{\prime}(0+)=0$. We define the sequence $\left(a_{k}, b_{k}\right)_{k \geq 0}$ as in (3.8) and follow the same notation as in Theorem A.1. We take $0<\eta<\alpha$ and $d_{p}>1$ such that $-\theta^{-} / d_{p}<p$ and $\theta^{-}<-\eta<\eta+p<\theta^{+}$, and let

$$
(\eta, \kappa, \vartheta)=\left(\eta, \frac{-\theta^{-}}{d_{p}}, p\right)
$$

Next, we verify the moment conditions of Theorem A.1 for the couple $\left(a_{0}, b_{0}\right)$. From the definition of $\left(a_{0}, b_{0}\right)$, we get

$$
\mathbb{E}\left[\ln a_{0}\right]=\frac{\psi^{\prime}(0+)}{q}=0, \quad \mathbb{E}\left[a_{0}^{\kappa}\right]=e^{\psi(-\kappa) / q} \quad \text { and } \quad \mathbb{E}\left[a_{0}^{-\eta}\right]=e^{\psi(\eta) / q},
$$

which are well defined. Similarly as in (1.5), by $L_{1}$-Doob's inequality (see Acciaio et al., 2013) and the Esscher transform (1.4)

$$
\mathbb{E}\left[b_{0}^{\eta}\right] \leq q^{\eta} \mathbb{E}\left[\sup _{0 \leq u \leq 1 / q} e^{-\eta \xi_{u}}\right] \leq \frac{e}{e-1} q^{\eta} e^{\frac{\psi(-\eta)}{q}}\left(1-\eta \psi^{\prime}(-\eta)-\psi(-\eta)\right)<\infty,
$$

and

$$
\mathbb{E}\left[a_{0}^{-\eta} b_{0}^{-\vartheta}\right] \leq q^{\vartheta} \mathbb{E}\left[e^{\eta \xi_{\frac{1}{q}}} \sup _{0 \leq u \leq 1 / q} e^{\vartheta \xi_{u}}\right] \leq q^{\vartheta} \mathbb{E}\left[\sup _{0 \leq u \leq 1 / q} e^{(\eta+\vartheta) \xi_{u}}\right]<\infty
$$

Therefore the asymptotic behaviour of $\mathcal{E}_{F}\left(q_{n}\right)$ for large $n$ follows from a direct application of Theorem A.1. In other words, there exists a positive constant $c(q)$ such that

$$
\sqrt{n} \mathcal{E}_{F}\left(q_{n}\right) \sim c(q), \quad \text { as } \quad n \rightarrow \infty .
$$

In order to get our result, we take $t$ to be a positive real number. Since the mapping $s \mapsto \mathcal{E}_{F}(s)$ is non-increasing, we get

$$
\sqrt{t} \mathcal{E}_{F}(t) \leq \sqrt{t} \mathcal{E}_{F}(\lfloor q t\rfloor / q)=\sqrt{\frac{t}{\lfloor q t\rfloor}} \sqrt{\lfloor q t\rfloor} \mathcal{E}_{F}(\lfloor q t\rfloor / q)
$$

Similarly

$$
\sqrt{t} \mathcal{E}_{F}(t) \geq \sqrt{t} \mathcal{E}_{F}((\lfloor q t\rfloor+1) / q)=\sqrt{\frac{t}{\lfloor q t\rfloor+1}} \sqrt{\lfloor q t\rfloor+1} \mathcal{E}_{F}((\lfloor q t\rfloor+1) / q) .
$$

Therefore

$$
\sqrt{t} \mathcal{E}_{F}(t) \sim c(q) q^{-1 / 2}, \quad \text { as } t \rightarrow \infty
$$


Moreover, we deduce that $c(q) q^{-1 / 2}$ is positive and does not depend on $q$. Hence we denote this constant by $c_{1}$. This concludes the proof of point ii).

iii) For the rest of the proof, we assume that $\psi^{\prime}(0)<0$. We first prove part a). Since $\psi^{\prime}(p)<0$, from Theorem 1.1 part iii)-a) we know that

$$
\mathbb{E}\left[I_{t}(\xi)^{-p}\right] \sim e^{t \psi(p)} \mathbb{E}^{(p)}\left[I_{\infty}(-\xi)^{-p}\right], \quad \text { as } t \rightarrow \infty .
$$

Hence the asymptotic behaviour is proven if we show that

$$
\mathcal{E}_{F}(t) \sim \mathbf{k} \mathbb{E}\left[I_{t}(\xi)^{-p}\right], \quad \text { as } \quad t \rightarrow \infty .
$$

Since $\psi^{\prime}(p)<0$, there is $\varepsilon>0$ such that $p(1+\varepsilon)<\theta^{+}, \psi(p(1+\varepsilon))<\psi(p)$ and $\psi^{\prime}((1+\varepsilon) p)<0$. Hence, from Lemma 2 (see the Appendix), we deduce that there is a constant $M$ such that

$$
\left|F\left(I_{t}(\xi)\right)-\mathbf{k} I_{t}(\xi)^{-p}\right| \leq M I_{t}(\xi)^{-(1+\varepsilon) p} .
$$

In other words, it is enough to prove

$$
\mathbb{E}\left[I_{t}(\xi)^{-(1+\varepsilon) p}\right]=o\left(e^{t \psi(p)}\right), \quad \text { as } \quad t \rightarrow \infty .
$$

From the Esscher transform (1.4) with $\lambda=(1+\varepsilon) p$, we deduce

$$
\begin{aligned}
\mathbb{E}\left[I_{t}(\xi)^{-(1+\varepsilon) p}\right] & =\mathbb{E}\left[e^{p(1+\varepsilon) \xi_{s}} I_{t}(-\xi)^{-(1+\varepsilon) p}\right] \\
& =e^{t \psi(p)} e^{t \psi_{p}(\varepsilon p)} \mathbb{E}^{((1+\varepsilon) p)}\left[I_{t}(-\xi)^{-(1+\varepsilon) p}\right] .
\end{aligned}
$$

This and Equation (1.5) with $\lambda=(1+\varepsilon) p$ imply that $\mathbb{E}^{((1+\varepsilon) p)}\left[I_{t}(-\xi)^{-(1+\varepsilon) p}\right]$ is finite for all $t>0$. Similarly as in the proof of Theorem 1.1 iii)-a), we can deduce that $\mathbb{E}^{((1+\varepsilon) p)}\left[I_{t}(-\xi)^{-(1+\varepsilon) p}\right]$ has a finite limit, as $t$ goes to $\infty$. We conclude by observing that $\psi_{p}(\varepsilon p)$ is negative implying that (3.10) holds. We complete the proof of point iii)-a) by observing that (3.9) and (3.10) yield

$$
\mathbb{E}\left[F\left(I_{t}(\xi)\right)\right] \sim \mathbf{k} \mathbb{E}\left[I_{t}(\xi)^{-p}\right], \quad t \rightarrow \infty .
$$

We now prove part b). Since $\psi^{\prime}(p)=0$ and $\psi^{\prime \prime}(p)<\infty$, from Theorem 1.1 part iii)-b) we know that there exists a positive constant $c_{2}$ such that

$$
\mathbb{E}\left[I_{t}(\xi)^{-p}\right] \sim c_{2} t^{-1 / 2} e^{t \psi(p)}, \quad \text { as } t \rightarrow \infty .
$$

Similarly as in the proof of part a), the asymptotic behaviour is proven if we show that

$$
\mathcal{E}_{F}(t) \sim \mathbf{k} \mathbb{E}\left[I_{t}(\xi)^{-p}\right], \quad \text { as } \quad t \rightarrow \infty,
$$

which amounts to showing that

$$
\mathbb{E}\left[I_{t}(\xi)^{-(1+\varepsilon) p}\right]=o\left(t^{-1 / 2} e^{t \psi(p)}\right), \quad \text { as } \quad t \rightarrow \infty
$$

for $\varepsilon$ small enough. The latter follows from Theorem 1.1 iii)-c).

Finally, we prove part c). Similarly as in the proof of part ii), we define the sequence $\left(a_{k}, b_{k}\right)_{k \geq 0}$ as in (3.8) and follow the same notation as in Theorem A.1. Let us choose $0<\eta<\alpha$ such that $0<\tau-\eta<\tau+p+\eta<\theta^{+}$and take

$$
(\eta, \kappa, \vartheta)=(\eta, \tau, p) \text {. }
$$


Next, we apply the Esscher transform (1.4) with $\lambda=\tau$ and observe

$$
\mathbb{E}\left[F\left(I\left(q_{n}\right)\right)\right] e^{-q_{n} \psi(\tau)}=\mathbb{E}^{(\tau)}\left[e^{-\tau \xi_{q_{n}}} F\left(I\left(q_{n}\right)\right)\right]=\mathbb{E}^{(\tau)}\left[A_{n}^{\tau} F\left(B_{n}\right)\right] .
$$

Hence in order to apply Theorem A.1, we need the moment conditions on $\left(a_{0}, b_{0}\right)$ to be satisfied under the probability measure $\mathbb{P}^{(\tau)}$. We first observe,

$$
\mathbb{E}^{(\tau)}\left[\ln a_{0}\right]=\mathbb{E}^{(\tau)}\left[\xi_{1 / q}\right]=e^{-\psi(\tau) / q} \mathbb{E}\left[\xi_{1 / q} e^{\tau \xi_{1 / q}}\right]=\frac{\psi^{\prime}(\tau)}{q}=0 .
$$

Similarly, we get

$$
\mathbb{E}^{(\tau)}\left[a_{0}^{\kappa}\right]=\mathbb{E}^{(\tau)}\left[e^{-\kappa \xi_{1 / q}}\right]=e^{-\psi(\tau) / q} \quad \text { and } \quad \mathbb{E}^{(\tau)}\left[a_{0}^{-\eta}\right]=\mathbb{E}^{(\tau)}\left[e^{\eta \xi_{1 / q}}\right]=e^{\psi_{\tau}(\eta) / q},
$$

where $\psi_{\tau}(\lambda)=\psi(\tau+\lambda)-\psi(\tau)$. From our assumptions both expectations are finite.

Again, we use similar arguments as those used in (1.5) to deduce

$$
\mathbb{E}^{(\tau)}\left[b_{0}^{\eta}\right] \leq q^{-\eta} \mathbb{E}^{(\tau)}\left[\sup _{0 \leq u \leq 1 / q} e^{-\eta \xi_{u}}\right] \leq q^{-\eta} e^{-\psi(\tau) / q} \mathbb{E}\left[\sup _{0 \leq u \leq 1} e^{(\tau-\eta) \xi_{u}}\right]<\infty,
$$

and

$$
\begin{aligned}
\mathbb{E}^{(\tau)}\left[a_{0}^{-\eta} b_{0}^{-p}\right] & \leq q^{p} \mathbb{E}^{(\tau)}\left[e^{\eta \xi_{\frac{1}{q}}} \sup _{0 \leq u \leq 1 / q} e^{p \xi_{u}}\right] \\
& \leq q^{p} e^{-\psi(\tau) / q} \mathbb{E}\left[\sup _{0 \leq u \leq 1} e^{(\tau+\eta+p) \xi_{u}}\right]<\infty .
\end{aligned}
$$

Therefore the asymptotic behaviour of $\mathbb{E}^{(\tau)}\left[A_{n}^{\tau} F\left(B_{n}\right)\right]$ follows from a direct application of Theorem A.1 with the functions $\tilde{\psi}(x)=F(x)$ and $\tilde{\phi}(x)=x^{\tau}$. In other words, we conclude that there exists a positive constant $c(q)$ such that

$$
n^{3 / 2} \mathbb{E}^{(\tau)}\left[A_{n}^{\tau} F\left(B_{n}\right)\right] \sim c(q), \quad n \rightarrow \infty .
$$

In particular from (3.11), we deduce

$$
\mathcal{E}_{F}\left(q_{n}\right) \sim c(q) e^{-n \psi(\tau) / q} n^{-3 / 2}, \quad n \rightarrow \infty .
$$

Then using the monotonicity of $F$ at infinity as in the proof of part ii), we get that for $n$ large enough,

$$
c(q) q^{-3 / 2} e^{-\psi(\tau) / q} \leq n^{3 / 2} e^{n \psi(\tau)} \mathcal{E}_{F}(n) \leq c(q) q^{-3 / 2} .
$$

A direct application of Lemma A.3 then yields the existence of a non-negative constant $c_{4}$ such that

$$
\lim _{q \rightarrow \infty} c(q) q^{-3 / 2}=C_{3} .
$$

Moreover, (3.12) yields that $C_{3}$ is positive. This ends the proof.

\section{A. Appendix}

We recall in this section a Theorem due to Guivarc'h and Liu (see Theorem 2.1 in Guivarc'h and Liu, 2001) and two technical Lemmas stated in Bansaye et al. (2013): 
Theorem A.1 (Guivarc'h and Liu, 2001). Let $\left(a_{n}, b_{n}\right)_{n>0}$ be $a \mathbb{R}_{+}^{2}$-valued sequence of i.i.d. random variables such that $\mathbb{E}\left[\ln a_{0}\right]=0$. Assume that $b_{0} /\left(1-a_{0}\right)$ is not constant a.s. and define

$$
A_{0}:=1, \quad A_{n}:=\prod_{k=0}^{n-1} a_{k} \quad \text { and } \quad B_{n}:=\sum_{k=0}^{n-1} A_{k} b_{k}, \quad \text { for } \quad n \geq 1 .
$$

Let $\eta, \kappa, \vartheta$ be three positive numbers such that $\kappa<\vartheta$, and $\tilde{\phi}$ and $\tilde{\psi}$ be two positive continuous functions on $\mathbb{R}_{+}$such that they do not vanish and for a constant $C>0$ and for every $a>0, b \geq 0, b^{\prime} \geq 0$, we have

$$
\tilde{\phi}(a) \leq C a^{\kappa}, \quad \tilde{\psi}(b) \leq \frac{C}{(1+b)^{\vartheta}}, \quad \text { and } \quad\left|\tilde{\psi}(b)-\tilde{\psi}\left(b^{\prime}\right)\right| \leq C\left|b-b^{\prime}\right|^{\eta} .
$$

Moreover, assume that

$$
\mathbb{E}\left[a_{0}^{\kappa}\right]<\infty, \quad \mathbb{E}\left[a_{0}^{-\eta}\right]<\infty, \quad \mathbb{E}\left[b_{0}^{\eta}\right]<\infty \quad \text { and } \quad \mathbb{E}\left[a_{0}^{-\eta} b_{0}^{-\vartheta}\right]<\infty .
$$

Then, there exist two positive constants $c(\tilde{\phi}, \tilde{\psi})$ and $c(\tilde{\psi})$ such that

$$
\lim _{n \rightarrow \infty} n^{3 / 2} \mathbb{E}\left[\tilde{\phi}\left(A_{n}\right) \tilde{\psi}\left(B_{n}\right)\right]=c(\tilde{\phi}, \tilde{\psi}) \quad \text { and } \quad \lim _{n \rightarrow \infty} n^{1 / 2} \mathbb{E}\left[\tilde{\psi}\left(B_{n}\right)\right]=c(\tilde{\psi}) .
$$

Lemma A.2. Assume that $F$ satisfies one of the Assumptions (A1) or (A2). Then there exist two positive finite constants $\eta$ and $M$ such that for all $(x, y)$ in $\mathbb{R}_{+}^{2}$ and $\varepsilon$ in $[0, \eta]$,

$$
\begin{aligned}
\left|F(x)-\mathbf{k} x^{-p}\right| & \leq M x^{-(1+\varepsilon) p}, \\
|F(x)-F(y)| & \leq M\left|x^{-p}-y^{-p}\right| .
\end{aligned}
$$

Lemma A.3. Assume that the non-negative sequences $\left(a_{n, q}\right)_{(n, q) \in \mathbb{Z}_{+}^{2}},\left(a_{n, q}^{\prime}\right)_{(n, q) \in \mathbb{Z}_{+}^{2}}$ and $\left(b_{n}\right)_{n \in \mathbb{Z}_{+}}$satisfy for every $(n, q) \in \mathbb{Z}_{+}^{2}$ :

$$
a_{n, q} \leq b_{n} \leq a_{n, q}^{\prime},
$$

and that there exist three sequences $(a(q))_{q \in \mathbb{Z}_{+}},\left(c^{-}(q)\right)_{q \in \mathbb{Z}_{+}}$and $\left(c^{+}(q)_{q \in \mathbb{Z}_{+}}\right.$such that

$\lim _{n \rightarrow \infty} a_{n, q}=c^{-}(q) a(q), \quad \lim _{n \rightarrow \infty} a_{n, q}^{\prime}=c^{+}(q) a(q), \quad$ and $\quad \lim _{q \rightarrow \infty} c^{-}(q)=\lim _{q \rightarrow \infty} c^{+}(q)=1$.

Then there exists a non-negative constant a such that

$$
\lim _{q \rightarrow \infty} a(q)=\lim _{n \rightarrow \infty} b_{n}=a .
$$

\section{Acknowledgements}

CS thanks the Centro de Investigación en Matemáticas, where part of this work was done. This work was partially funded by the Chair "Modélisation Mathématique et Biodiversité" of VEOLIA-Ecole Polytechnique-MNHN-F.X. and by the franco-mexican project PICS (CNRS) "Structures Markoviennes Auto-Similaires". SP and JCP acknowledge support from the Royal Society and SP also acknowledges support from CONACyT-MEXICO Grant 351643. The authors would like to thank anonymous referees for their comments and valuable suggestions that improved the exposition of the manuscript. 


\section{References}

B. Acciaio, M. Beiglböck, F. Penkner, W. Schachermayer and J. Temme. A trajectorial interpretation of Doob's martingale inequalities. Ann. Appl. Probab. 23 (4), 1494-1505 (2013). MR3098440.

V. I. Afanasyev, C. Böinghoff, G. Kersting and V. A. Vatutin. Limit theorems for weakly subcritical branching processes in random environment. J. Theoret. Probab. 25 (3), 703-732 (2012). MR2956209.

V. I. Afanasyev, J. Geiger, G. Kersting and V. A. Vatutin. Criticality for branching processes in random environment. Ann. Probab. 33 (2), 645-673 (2005). MR2123206.

V. Bansaye. Surviving particles for subcritical branching processes in random environment. Stochastic Process. Appl. 119 (8), 2436-2464 (2009). MR2532207.

V. Bansaye and J. Berestycki. Large deviations for branching processes in random environment. Markov Process. Related Fields 15 (4), 493-524 (2009). MR2598126.

V. Bansaye, J. C. Pardo Millan and C. Smadi. On the extinction of continuous state branching processes with catastrophes. Electron. J. Probab. 18, no. 106, 31 (2013). MR3151726.

V. Bansaye and F. Simatos. On the scaling limits of Galton-Watson processes in varying environments. Electron. J. Probab. 20, no. 75, 36 (2015). MR3371434.

J; Bertoin. Lévy processes, volume 121 of Cambridge Tracts in Mathematics. Cambridge University Press, Cambridge (1996). ISBN 0-521-56243-0. MR1406564.

J. Bertoin, A. Lindner and R. Maller. On continuity properties of the law of integrals of Lévy processes. In Séminaire de probabilités XLI, volume 1934 of Lecture Notes in Math., pages 137-159. Springer, Berlin (2008). MR2483729.

J. Bertoin and M. Yor. Exponential functionals of Lévy processes. Probab. Surv. 2, 191-212 (2005). MR2178044.

C. Böinghoff, E. E. Dyakonova, G. Kersting and V. A. Vatutin. Branching processes in random environment which extinct at a given moment. Markov Process. Related Fields 16 (2), 329-350 (2010). MR2666857.

C. Böinghoff and M. Hutzenthaler. Branching diffusions in random environment. Markov Process. Related Fields 18 (2), 269-310 (2012). MR2985138.

Th. Brox. A one-dimensional diffusion process in a Wiener medium. Ann. Probab. 14 (4), 1206-1218 (1986). MR866343.

P. Carmona, F. Petit and M. Yor. On the distribution and asymptotic results for exponential functionals of Lévy processes. In Exponential functionals and principal values related to Brownian motion, Bibl. Rev. Mat. Iberoamericana, pages 73-130. Rev. Mat. Iberoamericana, Madrid (1997). MR1648657.

R. A. Doney. Fluctuation theory for Lévy processes, volume 1897 of Lecture Notes in Mathematics. Springer, Berlin (2007). ISBN 978-3-540-48510-0; 3-540-48510-4. MR2320889.

R. M. Dudley. Real analysis and probability, volume 74 of Cambridge Studies in Advanced Mathematics. Cambridge University Press, Cambridge (2002). ISBN 0-521-00754-2. MR1932358.

S. N. Evans, A. Hening and S. J. Schreiber. Protected polymorphisms and evolutionary stability of patch-selection strategies in stochastic environments. Journal of mathematical biology pages 1-35 (2014). DOI: 10.1007/s00285-014-0824-5. 
Z. Fu and Z. Li. Stochastic equations of non-negative processes with jumps. Stochastic Process. Appl. 120 (3), 306-330 (2010). MR2584896.

J. Geiger, G. Kersting and V. A. Vatutin. Limit theorems for subcritical branching processes in random environment. Ann. Inst. H. Poincaré Probab. Statist. 39 (4), 593-620 (2003). MR1983172.

Y. Guivarc'h and Q. Liu. Propriétés asymptotiques des processus de branchement en environnement aléatoire. C. R. Acad. Sci. Paris Sér. I Math. 332 (4), 339-344 (2001). MR1821473.

K. Hirano. Determination of the limiting coefficient for exponential functionals of random walks with positive drift. J. Math. Sci. Univ. Tokyo 5 (2), 299-332 (1998). MR1633937.

K. Kawazu and H. Tanaka. On the maximum of a diffusion process in a drifted Brownian environment. In Séminaire de Probabilités, XXVII, volume 1557 of Lecture Notes in Math., pages 78-85. Springer, Berlin (1993). MR1308554.

T. G. Kurtz. Diffusion approximations for branching processes. In Branching processes (Conf., Saint Hippolyte, Que., 1976), volume 5 of Adv. Probab. Related Topics, pages 269-292. Dekker, New York (1978). MR517538.

A. Kuznetsov, J. C. Pardo and M. Savov. Distributional properties of exponential functionals of Lévy processes. Electron. J. Probab. 17, no. 8, 35 (2012). MR2878787.

A. E. Kyprianou. Introductory lectures on fluctuations of Lévy processes with applications. Universitext. Springer-Verlag, Berlin (2006). ISBN 978-3-540-31342-7; 3-540-31342-7. MR2250061.

A. E. Kyprianou and J. C. Pardo. Continuous-state branching processes and selfsimilarity. J. Appl. Probab. 45 (4), 1140-1160 (2008). MR2484167.

$\mathrm{Z}$. Li and W. Xu. Asymptotic results for exponential functionals of Lévy processes. ArXiv Mathematics e-prints (2016). arXiv: 1601.02363.

S. Palau and J. C. Pardo. Branching processes in a Lévy random environment. ArXiv Mathematics e-prints (2015). arXiv: 1512.07691.

S. Palau and J. C. Pardo. Continuous state branching processes in random environment: The Brownian case (2016+). To appear in Stoc. Proc, Appl.

J. C. Pardo, V. Rivero and K. van Schaik. On the density of exponential functionals of Lévy processes. Bernoulli 19 (5A), 1938-1964 (2013). MR3129040.

Ken-iti Sato. Lévy processes and infinitely divisible distributions, volume 68 of Cambridge Studies in Advanced Mathematics. Cambridge University Press, Cambridge (2013). ISBN 978-1-107-65649-9. MR3185174. 